\title{
Das Alter ehren: Vorstellungen vom Alter und Sorge um die Alten im Alten Orient
}

\author{
Stefan M. Maul
}

Adad-guppi ${ }^{1}$, eine Priesterin des Mondgottes von Harran, weiß in einer Inschrift aus dem 6. Jh. v. Chr. zu berichten, dass sie im Jahre 610 v. Chr. als Vierzigjährige die Zerstörung der letzten Feste des Assyrerreiches, der im Norden Syriens gelegenen assyrischen Stadt Harran, miterlebte. Über Jahrzehnte hinweg bis in ihr 95. Lebensjahr habe sie Sin, den Mondgott, angefleht, seinen Zorn zu besänftigen und endlich zuzulassen, dass sein Tempel in Harran wiedererrichtet würde. Schließlich, so Adad-guppi, habe der Gott sie erhört, indem er ihren leiblichen Sohn Nabonid (Regierungszeit 556-539 v. Chr.) zum babylonischen König erhob und diesen den Tempel in all seiner Pracht wiederaufbauen ließ:

\begin{abstract}
„Aus Liebe zu mir, die ich seine Gottheit verehrte“, heißt es in der Inschrift, „(...) erhöhte Sin, der König der Götter, mein Haupt und verlieh mir einen guten Namen im Lande. Lange Tage, Jahre voll Herzensfreude gab er mir dazu. Von der Zeit des Assurbanipal, des Königs von Assyrien, bis zum 9. Jahr des Nabonid, des Königs von Babylon, meines leiblichen Sohns, hielt mich Sin, der König der Götter, 104 gütige Jahre lang am Leben in der Ehrfurcht, die er mir ins Herz legte. Der Blick meiner Augen war hell, außergewöhnlich gut mein Gehör, Hände und Füße waren gesund, erlesen meine Worte. Essen und Trinken schmeckte mir, meine Gesundheit war gut und froh mein Herz. Meine KindesKindes-Kindes-Kinder - gesund bis in die 4. Generation - erlebte ich, während ich mich an (meinem) gesegneten Lebensalter sättigte. “2
\end{abstract}

Für den Historiker gibt es keinen ersichtlichen Grund, Zweifel daran zu hegen, dass die Mutter des letzten babylonischen Königs tatsächlich in körperlicher und geistiger Gesundheit ihren 100. Geburtstag noch um Jahre überlebte. ${ }^{3}$

Denn auch vor zwei, drei und mehr Jahrtausenden verfügte der Mensch - nicht anders als wir - über seine grundlegende biologische Beschaffenheit, welche es ihm erlaubt, unter besonders glücklichen Bedingungen das elfte oder gar das zwölfte

\footnotetext{
${ }^{1} \mathrm{Zu}$ der Person Adad-guppi sowie der Deutung und der korrekten Lesung ihres Namens siehe Schaudig, H. (2001) 14.

${ }^{2}$ Adad-guppi-Inschrift, Kolumne II:22-34 (zitiert nach Hecker, K. (1988) 482-483; siehe auch Schaudig, H. (2001) 500-513).

${ }^{3}$ Tatsächlich gibt es Anlaß zu der Vermutung, dass Adad-guppi lediglich 102 Jahre alt wurde (Schaudig, H. (2001) 14 mit Anm. 42).
} 
Lebensjahrzehnt zu erreichen. Das Wissen anderer, weit vor uns liegender Zeitalter um die Möglichkeit des Menschen, tatsächlich mehr als hundert Jahre alt zu werden, findet etwa in der Genesis, dem ersten Buch der hebräischen Bibel, seinen Ausdruck (Gn 6:1-4). Dort heißt es nämlich, Gott habe nach der Sintflut den zuvor sehr viel längeren Lebenshorizont des Menschen auf maximal 120 Jahre begrenzt.

Zwar sorgte im Alten Orient, von dem hier die Rede sein soll ${ }^{4}$ - ebenso wie in vielen anderen vormodernen Gesellschaften - eine enorm hohe Kindersterblichkeit für eine durchschnittliche Lebenserwartung, die drastisch unter der heutigen lag (Böck, B., 2000, 30-31; Dandamaev, M.A., 1980). Gleichwohl gab es die Alten. Und entgegen den weitverbreiteten Vorstellungen von Verhältnissen in den frühen Perioden der menschlichen Zivilisation rechneten auch die antiken Kulturen mit einer beachtlich hohen Lebenserwartung, zumindest für jene, die das Erwachsenenalter bereits erreicht hatten. So gibt etwa der Psalmist in Psalm 90:10 folgende Einschätzung ${ }^{5}$ :

„Unser Leben währt siebzig Jahre, und wenn es hoch kommt, sind es achtzig. Das Beste daran ist nur Mühsal und Beschwer, rasch geht es vorbei, wir fliegen dahin.“

Auch der griechische Historiker Herodot gelangte im 5. vorchristlichen Jahrhundert zu einem ähnlichen Schluss. „Auf siebzig Jahre setze ich die Dauer des Menschenlebens", lässt er Solon in einem Dialog mit Kroisos sagen. ${ }^{6}$ Einer babylonischen Lehre zufolge galt ebenfalls ein Alter von zumindest 60-70 Jahren als das, was ein Mensch gerechterweise erwarten darf. In einem als Gelehrtengeheimnis bezeichneten Text aus dem 7. Jh. v. Chr. heißt es nämlich:

,(Das Alter von) vierzig (Jahren ist des Menschen) Blüte. Fünfzig (Jahre wären) kurze Tage.

Sechzig (Jahre sind) das Mannesalter, siebzig (Jahre) lange Tage, achtzig (Jahre) sind die (Lebensjahre) der Weisen, neunzig (Jahre) sind ein gesegnetes Alter.“7

Freilich sorgten die vergleichsweise begrenzten Möglichkeiten der Heilkunde, akut lebensbedrohliche Krankheitszustände erfolgreich zu bekämpfen, dafür, dass die Anzahl alter Leute und namentlich der gebrechlichen deutlich geringer war, als das heute der Fall ist. ${ }^{8}$ So sind in der sehr umfangreichen Literatur der Keilschriftkulturen zwar bisweilen die Leiden des Alters beschrieben (Heeßel, N.P., 2000, 72 mit Anm. 19). Unter den kaum überschaubaren heilkundlichen Traktaten, die der Alte Orient in mehr als zwei Jahrtausenden hervorgebracht hat, finden sich jedoch bezeichnenderweise zahlreiche Anweisungen zur Heilung von Säuglingen, Kleinkindern und Frauen. Aber wir kennen nicht einen einzigen Text therapeutischen oder pharmakologischen Inhalts, der explizit den Leiden des Alters gewidmet wäre. Wer im höheren Alter schwer krank wurde, dürfte in der Regel rasch gestorben sein.

In der schriftlichen Überlieferung fehlen uns Klagen über den Tod eines reiferen oder alten Menschen. Allein das Sterben zur Unzeit, d. h. der Tod derjenigen, die

\footnotetext{
${ }^{4}$ An dieser Stelle sei auf die grundlegende Publikation verwiesen, von der der vorliegende Aufsatz maßgeblich profitiert hat: Stol, Vleeming 1998. Siehe außerdem Greenfield, J.C. (1982).

${ }^{5}$ Siehe auch Malamat, A. (1982).

${ }^{6}$ Herodot, 1, 32 (zitiert nach Feix, J. (1963) 31).

${ }^{7}$ Gurney, O.R. (1964) Text Nr. 400:45-47.

${ }^{8}$ Einen Überblick über die altorientalische Heilkunde gibt Geller, M.J. (2010).
} 
noch im jugendlichen Alter dahinschieden und weder einen Ehegatten noch Kinder gehabt hatten, galt als beklagenswert. Auf den gleichwohl vorhandenen Wunsch, in Gesundheit ein hohes Alter zu erreichen, treffen wir immer wieder, namentlich in Briefen. Ein schönes Beispiel sei hier vorgestellt. Es stammt aus einem Brief, den ein gelehrter Heiler im 7. Jh. v. Chr. an den König Assyriens richtete:

„Mögen (...) (alle) großen Götter des Himmels und der Erde, die Götter, die in Assyrien und die Götter, die in Babylonien wohnen, die Götter der Gesamtheit aller Länder, den König, meinen Herrn, in ganz besonderer Weise segnen. Dem König, meinem Herrn, mögen sie lange Tage, zahlreiche Jahre, Frohsinn und eine gute Konstitution, Gesundheit und Lebenskraft geben.

So wie Sonne und Mond am Himmel fest verankert sind, so möge die Herrschaft des Königs, meines Herrn, und die seiner Nachkommschaft in allen Ländern fest verankert sein. Das Gedeihen Assyriens, das Gedeihen Babyloniens und das Gedeihen aller Länder mögen sie (die Götter) den König, meinen Herrn, erblicken lassen. Sie mögen dem König, meinem Herrn, Frohsinn und eine gute Konstitution, das Aufleuchten seines Gemütes, Altwerden bis in ferne Tage und eine sehr lange Amtszeit schenken. “9

In einem anderen Brief ist von den noch kleinen Kindern des Königs die Rede. Sie, so schreibt der Berater des Königs, mögen ruhig in dessen ,heilvollen Schatten spielen. “ Und er fügt hinzu: „Der König, mein Herr, möge sie gedeihen sehen. Und auch noch deren Enkelkinder mögen in gleicher Weise in der Gegenwart des König, meines Herrn spielen. “10

In einem Bericht, den er an den assyrischen König sandte, wünschte ein Astrologe sogar der König möge das Alter des sagenhaften ersten vorsintflutlichen Königs Alulim erreichen, ${ }^{11}$ von dem es hieß, er sei 36000 Jahre alt geworden. ${ }^{12}$

Ähnlich wie in der biblischen Kultur lehrten nämlich auch Babylonier und Assyrer, dass Tod, Alter und Gebrechlichkeit keineswegs von Anbeginn an in der Welt gewesen seien. Im Anfang nämlich hat es alten mesopotamischen Schöpfungsmythen zufolge Tod durch Altersschwäche gar nicht gegeben. So erreichten die Menschen die eben genannten, unvorstellbar langen Lebenszeiten. Sie vermehrten und vermehrten sich und wurden mit den Jahrzehntausenden so zahlreich, dass ihr geschäftiges Treiben, ihr Geschrei nicht nur die Götter in ihrer Ruhe störte, sondern auch die Fähigkeit des Menschen übertönte, Gottes Stimme zu hören. So beschlossen die Götter eine große Flut zu schicken, auf dass die Menschheit vernichtet werde. ${ }^{13}$ Allein der Weisheitsgott verriet den Plan Uta-napischti, dem babylonischen Noah, der mit seiner Familie als einziger der alles vernichtenden Flut mit Hilfe einer nach genauen Vorgaben angefertigten Arche entkam, in der - auf weisheitsgöttlichen Rat - auch die Tiere das urzeitliche Weltengericht überlebt hatten. In der neuen, auf Uta-napischti und die Seinen zurückgehenden Welt sollte Vergleichba-

\footnotetext{
${ }^{9}$ Parpola, S. (1993) 160, Text Nr. 197:7ff.

${ }^{10}$ Parpola, S. (1993) 166, Text Nr. 207:r.3ff.

11 Parpola, S. (1993) 120, Text Nr. 158:4.

${ }^{12} \mathrm{Zu}$ König Alulim, der auch Alulu genannt wurde und 36000 Jahre (einer anderen Tradition zufolge 28800 Jahre) lang regiert haben soll, sind alle uns bekannten Informationen und die einschlägigen weiterführenden Literaturverweise zusammengestellt in Frahm, E. (2009) 141.

${ }^{13} \mathrm{Zu}$ den Sintflutgeschichten des Alten Orients siehe Maul, S.M. (2007) und Maul, S.M. (2008) $37-40$ und $140 \mathrm{ff}$.
} 
res nicht mehr passieren. Teil des Bundes, den die Götter nun mit den Menschen schlossen, war, dass diese von nun an eine ins menschliche Maß begrenzte Lebenszeit haben würden. Altersgebrechlichkeit und Tod galten so als Unterpfand dafür, dass es eine Sintflut nie mehr geben würde.

Mit der Bedürftigkeit alter Menschen sahen sich auch vor Jahrtausenden die Gesellschaften des Alten Orients konfrontiert. Wenn die Alten nicht mehr oder nur noch bedingt erwerbsfähig waren, galt es - nicht anders als heute - ihnen Einkommen, Ernährung, Kleidung und Unterkunft zu sichern; ihnen ein Feld sozialer Ansprache zu erhalten und im Krankheitsfall Pflege zukommen zu lassen und nicht zuletzt auch sicherzustellen, dass der Besitz einer alten kranken oder dementen Person sachgerecht verwaltet würde. Einer weiteren Sorge kam im alten Zweistromland ein weit größerer Stellenwert zu, als dies in den modernen westlichen Industriegesellschaften der Gegenwart der Fall ist: Es mußte sichergestellt werden, dass eine Person ordnungsgemäß bestattet und deren als unsterblich gedachte jenseitige Existenz auch weiterhin durch bestimmte Riten erhalten wurde. ${ }^{14}$

In unserer Gegenwartsgesellschaft sind diese Aufgaben mehr und mehr dem Staat zugefallen. Im Alten Zweistromland oblag hingegen die Sorge um die Alten ausschließlich der Familie. Familiengründung war daher - zumindest für die frei Geborenen - eine soziale und ökonomische Notwendigkeit (Wilcke, 1984). Dies läßt sich auf die einfache Formel bringen: Wer Frau und Kind hat, dessen Altersversorgung ist gesichert.

Prosopographische Studien (Roth, M., 1987; van Driel, G., 1998, 167-168) haben gezeigt, dass in Babylonien und Assyrien über die Jahrhunderte hinweg - zumindest in wohlhabenden Familien - ein Mann üblicherweise mit Mitte bis Ende Zwanzig ein etwa 10-15 Jahre jüngeres Mädchen heiratete, welches nicht nur zumindest einen Sohn gebar, sondern - aufgrund des Altersunterschiedes - im Alter wohl auch für die Pflege ihres Mannes sorgen konnte. Gleichzeitig standen der Frau Kapitalrücklagen zur Verfügung, die beim Vorversterben des Ehemannes nicht zu dessen Nachlass gehörten, sondern im Besitz der Frau blieben. ${ }^{15}$ Die Sorge um die alte Mutter oblag dann dem Sohn.

Söhne zu haben, war daher schlicht eine Lebensnotwendigkeit. Dem medizinischen Problem der Kindersterblichkeit kam in der Heilkunde deshalb besonders große Aufmerksamkeit zu (Stol, M., 2000). Die fundamentale Existenzbedrohung, die in dem damals allzu häufigen Tod der kleinen Kinder lag, findet einen beredten Ausdruck in der Löwenfratze der blutgierigen Dämonin Lamaschtu (Farber, W., 1980-1983), in der die Kindersterblichkeit für die Mesopotamier eine darstellbare Gestalt gewann.

Eine Gilgamesch-Erzählung des dritten vorchristlichen Jahrtausends (George, A.R., 2003, 727-777) führt vor Augen, welch enorme Wertschätzung man dem Reichtum an Söhnen entgegenbrachte. In der folgenden Szene befragt Gilgamesch den der Unterwelt entstiegenen Geist seines toten Freundes Enkidu:

\footnotetext{
${ }^{14} \mathrm{Zu}$ Bestattungssitten und Totenpflege siehe Mofidi Nasrabadi, B. (1999) und Tsukimoto, A. (1985).

${ }^{15}$ Vgl. hierzu die exemplarische Untersuchung Roth, M. (1991/93).
} 
„Sahst du den, der einen einzigen Sohn hatte?“" „Ich habe ihn gesehen.

Der Kuckuck klebt an seinem Haus, und bitterlich weint er darüber.“

„Sahst du den, der zwei Söhne hatte?“ „Ich habe ihn gesehen.

Auf zwei Ziegeln sitzt er und ißt das Brot.“

„Sahst du den, der drei Söhne hatte?“ „Ich habe ihn gesehen.

Aus einem Schlauch, befestigt am Sattel, trinkt er das Wasser!“

„Sahst du den, der vier Söhne hatte?“ „Ich habe ihn gesehen.

Wie einer, der über angespannte Esel verfügt, ist froh sein Herz!“

„Sahst du den, der fünf Söhne hatte?“ „Ich habe ihn gesehen.

Seine Hand ist geschickt wie die eines guten Schreibers.

Den Palast betritt er mit Leichtigkeit.“

„Sahst du den, der sechs Söhne hatte?“” „Ich habe ihn gesehen.

Wie ein Landmann ist er frohen Herzens."

„Sahst du den, der sieben Söhne hatte?“ „Ich habe ihn gesehen.

Wie ein junger Bruder der Götter sitzt er auf einem Thron und lauscht der Musik!“

„Sahst du den, der keinen Erben hat?“ „Ich habe ihn gesehen.

Wie auf einem gebrannten Ziegel nagt er am Brot!“16

Noch im Jenseits ist nämlich der hier ausgebreiteten Vorstellung zufolge die alte Generation auf die Sorge der Jüngeren angewiesen. Die wechselseitige enge Verbundenheit der Elterngeneration mit der der Kinder war in den Gesellschaften des Alten Zweistromlandes lebenswichtig. Das Ehren der Eltern und des Alters war daher nicht nur ein immer wieder formuliertes moralisches Postulat. Auch im Alltag verlangte die streng eingehaltene Sitte, dass der Sohn dem Vater in Beruf und Besitz nachfolgte, Harmonie zwischen den Generationen. Denn selbst, wenn der Sohn die Geschäfte des Vaters führte, lag - solange der Valter lebte - Verantwortung und Entscheidungsbefugnis allein bei dem Alten. Dass man dennoch sicherstellte, dass die Generationen immer wieder zu einem für alle Beteiligten zufriedenstellenden Ergebnis gelangten, zeigen Herrschaftsformen, wie sie im Gilgamesch-Epos geschildert sein. Der selbstherrliche König kann dort nämlich keine Entscheidung von Gewicht fällen, ohne zuvor den Rat der Ältesten und den der jungen Krieger befragt zu haben, die zu einem Ausgleich gelangen müssen. ${ }^{17}$

Auch wenn wir aus dem Alten Orient keinerlei gesetzliche Regelungen zur Altenversorgung kennen, zeugt doch folgendes "Gesetz“ aus dem im 18. Jh. v. Chr. niedergeschriebenen Rechtsbuch des Hammurapi davon, dass man auch im frühen Babylonien eine moralische Pflicht darin sah, Kranke und Hilflose in ihrer Familie adäquat zu versorgen. In dem hier zitierten Paragraph des Kodex Hammurapi geht es um eine Ehefrau, die sich eine schlimme, ansteckende und unheilbare, la'bum genannte Krankheit zugezogen hatte:

„Wenn ein Mann eine Ehefrau nimmt und die la'bum-Krankheit sie befällt - wenn er dann plant, eine andere zu heiraten, so darf er das tun; seine (erste) Ehefrau, welche die la'bumKrankheit befallen hat, darf er (jedoch) nicht verstoßen; im Haushalt, den er aufgebaut hat, soll sie wohnen bleiben und, solange sie lebt, soll er sie unterhalten."18

${ }^{16}$ Gilgamesch-Epos, Tafel XII:102-116 (George, A.R. (2003) 732-735) und „Bilgames und die Unterwelt", Z. 255-a 2 (George, A.R. (2003) 760-764). Die hier vorgelegte Übersetzung stammt vom Verfasser.

${ }^{17}$ Siehe z. B. Maul, S.M. (2008) 26.

${ }^{18}$ Kodex Hammurapi $§ 148$ (Übersetzung nach Borger, R. (1982) 60). 
Kindern dürften man ihren alten und nicht mehr erwerbsfähigen Eltern gegenüber die gleiche Verantwortung abverlangt haben, die laut Kodex Hammurapi ein Ehemann für seine schwerkranke Gattin wahrzunehmen hatte. In dem Prolog eines Rechtsbuches aus dem 20. Jh. v. Chr. rühmt sich Lipit-Ischtar, der König der südmesopotamischen Stadt Isin, er habe in seiner Regierungszeit dafür gesorgt, dass , der Vater seine Kinder unterstützte (und) die Kinder ihren Vater unterstützen“ (Lutzmann, H., 1982, 25:17-20). Man mag daraus schließen, dass die Wirklichkeit von Altenpflege und Verantwortlichkeit der Generationen für einander doch nicht immer den oben herausgearbeiteten moralischen Anforderungen entsprach.

Eine Urkunde aus dem späten 6. vorchristlichen Jahrhundert gibt uns Einblick in die diesbezüglichen Nöte einer babylonischen Familie. Folgendermaßen ist ihr Wortlaut:

A. sagte zu seiner Tochter B.: „Als ich krank war, hat mich mein Bruder C. verlassen, und mein Sohn D. ist mir davongelaufen. Nimm mich bei dir auf und sorge für mich und gib mir Zuwendung an Nahrung, Öl und Kleidung, solange ich lebe. Und ich werde dir meine Pfründe... überschreiben." B. ging auf das Angebot ihres Vaters A. ein und nahm A. auf in ihrem Haus und gab ihm Zuwendung an Nahrung, Öl und Kleidung. A. überschrieb aus freiem Willen seine Pfründe ... mit gesiegelter Urkunde seiner Tochter B. auf ewig. Solange A. lebt, soll B. ihrem Vater A. Zuwendung an Nahrung, Öl und Kleidung geben. Solange A. lebt, soll er über das Einkommen aus seiner Pfründe verfügen können. Aber A. darf seine Pfründe weder verkaufen, noch verschenken noch verpfänden, noch davon etwas abziehen. Von dem Augenblick an, da A. tot ist, soll sie seiner Tochter B. überschrieben sein. ${ }^{19}$

In dem hier vorgestellten Fall war die Familiensituation offenbar so problematisch, dass die Lösung des sich abzeichnenden Problems, nämlich die Altersversorgung des alleingelassenen Vaters, einer vertraglichen Regelung bedurfte, die - um deren Durchsetzung sicher zu gewährleisten - Autoritäten bemühen mußte, welche außerhalb des Familienverbandes stehen. Und dies, obgleich doch der Vater A. mit der Pfründe über ein regelmäßiges Einkommen verfügte, das körperliche Arbeit nicht notwendig machte. Der Bruder als nächster Verwandter aus der eigenen Generation war der Erwartung, sich um den erkrankten Mann zu kümmern, nicht nachgekommen, der Sohn davongelaufen. Unserem babylonischen Vater blieb nur noch die Tochter. Doch was veranlaßte ihn, seine in die Hände der Tochter gegebene Altersversorgung auch vertraglich abzusichern? Mit dieser Frage ist auch die Frage verbunden, ob es im Alten Orient eine Art Unterhaltspflicht der Kinder gegeben hat, und wenn ja, wie diese geregelt war.

Schon anhand der oben besprochenen Urkunde ist deutlich zu erkennen, dass das Problem der Altersversorgung eng mit erbrechtlichen Regelungen verbunden war. Dies ist natürlich und auch naheliegend. Denn nur so kann Geben und Nehmen zwischen den Generationen in Einklang gebracht werden. Schauen wir also kurz auf die Grundregeln babylonischen Erbrechtes. ${ }^{20}$ Dort war es so, dass nur Söhne - und zwar ausschließlich die aus einer rechtmäßigen Ehe - erbberechtigt waren. Aus dem Erbrecht aber erwuchs den Söhnen eine Versorgungspflicht gegenüber den alten El-

${ }^{19}$ Ungnad, A. (1908) Text Nr. 21. Vgl. die Bearbeitung: San Nicolò, M. (1932) 44-46. Die hier vorgestellte Übersetzung richtet sich nach Westbrook, R. (1998) 11.

${ }^{20}$ Als Einführung seien empfohlen: Neumann, H. (2003) und Westbrook, R. (2003). 
tern. Dieser Regelfall, der einen Vertragsschluß zwischen Vater und Sohn bezüglich der Altersversorgung unnötig werden läßt, galt in der oben behandelten Situation aber nicht, da der Sohn zwar vorhanden, aber davongelaufen war. Zum Zweck der Absicherung des nicht regulären Erbrechtes der Tochter galt es also, eine rechtskräftige und einklagbare Regelung zu finden.

Die zahlreichen uns erhalten gebliebenen keilschriftlichen Urkunden aus drei Jahrtausenden, in denen dokumentiert ist, dass man mit Rechtsmitteln Altersversorgung zu regeln versuchte, erweisen sich dementsprechend als Regelungen, die vonnöten waren, da der Normalfall der Altersversorgung nicht verwirklicht werden konnte - sei es, weil ein Ehepaar ohne Sohn geblieben war; sei es, weil die Söhne verstorben, vermißt, verschleppt oder davongelaufen waren, oder sei es, weil die Söhne nicht willig oder fähig waren, ihre Eltern im Alter zu versorgen.

Drei Grundstrategien lassen sich ausmachen, den Mangel an Söhnen durch den Einsatz von Besitz auszugleichen.

Die erste und wohl auch wichtigste ist die schon seit dem dritten vorchristlichen Jahrtausend belegte Adoption, also die rechtlich abgesicherte Begründung eines Vater-Sohn-Verhältnisses ohne Rücksicht auf die biologische Abstammung. ${ }^{21}$ Recht häufig ist der Fall, dass ein kinderlos gebliebenes Ehepaar bereits in jüngeren Jahren einen Jungen an Sohnes Statt annahm, dem es eine Ausbildung zukommen ließ, und so dafür sorgte, dass rechtzeitig ein adäquater Erbe mit allen seinen Rechten und Pflichten heranwuchs. Wir kennen daneben - und dies nicht selten - aber auch Adoptionen, die zu einem späteren Zeitpunkt eigens zur Absicherung des Alters durchgeführt wurden. In diesen, in drei Jahrtausenden belegten Fällen, wird vor Zeugen und Gericht einer erwachsenen Person zugesichert, das Erbe des Adoptierenden so wie ein leiblicher Sohn anzutreten zu können, unter der Maßgabe, dass der Adoptierte die Altenpflege des Adoptierenden übernimmt. Ausführlichere Adoptionsurkunden dieser Art regeln neben der Pflicht zur Grundversorgung des Alten mit Getreide, Öl und Kleidung auch Einzelheiten wie z. B. den Anspruch des zu Versorgenden auf Fleisch an hohen Feiertagen. ${ }^{22}$ Nicht wenige Dokumente beziehen in die Pflichten des Versorgenden auch die Forderung ein, dem zu Betreuenden mit dem gebotenen Respekt ( palāhu) zu begegnen und ihn in jeder Hinsicht zu ehren ( $k u b-$ butu). ${ }^{23}$ Das hier verwendete Wort ist identisch mit dem hebräischen Verb, das für das „Ehren“ von Vater und Mutter in dem vierten der zehn Gebote steht. ${ }^{24}$ Mit den Begriffen palāhu und kubbutu sind „Hochachtung“ und ,liebevolle Zuwendung“ umschrieben. Manche keilschriftlichen Verträge fügen zu den oben genannten Bestimmungen noch hinzu, dass Respekt und Zuwendung so zu erbringen seien, dass der Betreute dabei ,frohen Herzens“ (Stol 1998, 62) sei.

Die zweite Strategie, auf die wir in den altorientalischen vertraglichen Regelungen zur Altersvorsorge immer wieder stoßen, greift anders als die erste nicht dau-

${ }^{21}$ Zur Adoption im Alten Orient siehe David, M. (1927); Neumann, H. (2003); Westbrook, R. (1993 und 2003).

${ }^{22}$ Siehe Westbrook, R. (1998) 10.

${ }^{23}$ Siehe Westbrook, R. (1998) 10; Stol, M. (1998) 62f. und Veenhof, K.R. (1998) 127ff.

${ }^{24}$ Zu diesem Verb ,ehren“ siehe Albertz, R. (1978). 
erhaft in eine Familienstruktur ein. Sie besteht nämlich darin, dass ein Sklave sich vor Zeugen verpflichtet, seinen Besitzer zu pflegen, während dieser seinem Diener im Gegenzug verbindlich zusagt, dass dessen Freilassung im Augenblick seines eigenen Todes erfolgt. ${ }^{25}$ Die Aussicht, vielleicht schon in wenigen Jahren gänzlich über die eigene Person verfügen zu können, wird namentlich den jüngeren Unfreien attraktiv erschienen sein. ${ }^{26}$ Wohlhabende alte Leute konnten sich auf diese Weise so wie etwa die aus reicher Familie stammende babylonische Nonne Innabatum im 19. Jh. v. Chr. - ein ganzes Pflegeteam zusammenstellen. Die alte, in einem Kloster lebende Dame hatte sich nämlich - wie wir aus entsprechenden Urkunden wissen - zur Pflege und Unterhaltung gleich drei Mädchen aus dem Besitz ihrer Familie verpflichtet, welche nach dem Hinscheiden der Nonne mit weit besseren Lebensbedingungen als bei ihren ursprünglichen Besitzern rechnen durften (Stol, M., 1998, 111-112).

Die dritte Strategie zur Absicherung des Alters, ohne hierfür auf eigene Söhne zurückgreifen zu müssen, bestand schließlich darin, Altenpflege durch Schuldtilgung einzukaufen. Eine Urkunde aus dem syrischen Emar, die im letzten Drittel des zweiten vorchristlichen Jahrtausends geschrieben wurde, führt uns einen solchen Fall vor Augen:
„A. sagte folgendermaßen: ,B. stand bei mir mit 41 Schekel Silber in der (dienstantichreti- schen) Schuld. Jetzt habe ich 20 Schekel von diesem Betrag gestrichen und ihm (außerdem) C. zur Frau gegeben. ' Solange A. und seine Frau D. leben, soll B. sie ehren. Wenn er sie ehrt, kann er, wenn sie verstorben sind, seine Frau und seine Kinder nehmen und gehen, wohin er will. Er wird die (noch ausstehenden) 21 Schekel unseren Kindern zurückzahlen."

In diesem Fall sind die Kinder des alten Ehepaares von der Last der Altenpflege aus welchen Gründen auch immer befreit. Nach dem Tode der Alten, so sieht es die Regelung vor, soll B. seine Restschuld bezahlen, oder aber den Kindern der Verblichenen weiterdienen. Darüber hinaus werden beide Parteien im Fall des vorzeitigen Abbruchs der Vereinbarung mit Vertragsstrafen belegt. Der Gläubiger soll seines Geldes verlustig gehen, der Schuldner, der seiner Pflegeverpflichtung nicht nachkommen sollte, soll hingegen die ursprüngliche Gesamtschuld zuzüglich eines Zinsbetrages von $50 \%$ an den Gläubiger zahlen. Auf diese Weise sind beide Vertragspartner fest an ihr Abkommen gebunden.

Die Möglichkeit, auf die eine oder die andere Weise, die eigene Altersversorgung mit Kapitalbesitz abzusichern, wenn eigene Kinder bzw. Söhne nicht vorhanden waren, stand freilich nur jenen offen, die entsprechendes Vermögen ihr eigen nannten. In allen Epochen der altorientalischen Geschichte dürfte diese reichere Gesellschaftsschicht nur eine kleine Minderheit gewesen sein. Die vielen anderen Menschen, die weder über nennenswerten Besitz noch über eigene Söhne verfügten, welche ihr Leben im Alter absichern konnten, blieben hingegen sich selbst und

\footnotetext{
${ }^{25}$ Vgl. die in Stol, M. (1998) 83 Anm. 95 hierzu zusammengestellte Literatur.

${ }^{26}$ Zum Phänomen des Personenbesitzes im Alten Orient siehe Neumann, H. (2003) und Westbrook, R. (2003).

${ }^{27}$ Arnaud, D. (1986) 24-26, Text Nr. 16:1-9, siehe dazu auch Westbrook, R. (2004) 104. Die hier gegebene Übersetzung richtet sich nach Westbrook, R. (1998) S. 18.
} 
dem Wohlwollen Dritter überlassen. Mangels Kapitals erübrigte es sich, ihre Altersversorgung vertraglich zu regeln. So ist ihr Schicksal meist nicht aktenkundig geworden und uns in der Regel verborgen geblieben.

Dennoch haben sich Dokumente erhalten, die uns auch Einblick in das Leben jener altgewordenen einfachen Leute geben können. Hierzu zählen Personalverzeichnisse, die in staatlichen Webereien geführt und im 21. vorchristlichen Jahrhundert, in der Zeit des Reiches der III. Dynastie von Ur, niedergeschrieben wurden. In diesen Listen sind die Arbeitskräfte, zumeist Frauen, namentlich aufgeführt. Manche der Namen, es sind etwa $6 \%$ des gesamten Personalbestandes, sind mit dem Zusatz šu-gi, ,Greis‘, versehen. Diesen alten Arbeiterinnen wurden Monatsrationen von 201 Gerste zugewiesen, ein Betrag, der sonst Kindern zusteht, und wohl nur gerade das Lebensminimum garantiert (Wilcke, C., 1998, 26ff.). In anderen Personallisten dieser Zeit sind auch alte Männer erwähnt. Diese ebenso mit sehr schmalen Rationen bedachten Alten verdingten sich als Fischer, Vogelfänger und Hilfsarbeiter (Wilcke, C., 1998, 36ff.). Wenn sie, wie nicht selten, auch dort im Familienverband arbeiteten, konnten ihre Kinder zu ihrer Versorgung beitragen. Standen keine Kinder zur Verfügung, galt es mit dem Wenigen auszukommen, das die jeweilige Institution gewährte.

\section{Zusammenfassung}

Die vorliegende Untersuchung zeigt, dass es auch vor Jahrtausenden in den untergegangenen Kulturen des Alten Vorderen Orients Alte gab, die sogar das 11. Lebensjahrzehnt erreichten. Zwar bildete sich ein eigener gerontologischer Zweig der ansonsten recht hoch entwickelten altorientalischen Heilkunde nicht aus, da schwere Krankheiten in hohem Lebensalter angesichts der letztlich begrenzten Möglichkeiten der Medizin wohl in der Regel rasch zum Tod führten. Wir können aber beobachten, dass die Gesellschaften des Alten Orients Normen entwickelten, die die Familie und namentlich die Söhne einer alten Person der strengen moralischen Verpflichtung unterwarfen, Alten, die nicht mehr oder nur bedingt erwerbsfähig waren, Einkommen und Ernährung, Kleidung, Unterkunft, soziale Ansprache und im Krankheitsfall auch Pflege zukommen zu lassen. Für den Fall, dass ein männlicher Erbe fehlte, der die Aufgabe der Versorgung der Alten übernehmen konnte, kennen wir aus allen Epochen der altorientalischen Geschichte, vom dritten vorchristlichen Jahrtausend bis zum Ende der Keilschriftkultur um die Zeitenwende, Keilschrifttexte, in denen Altersvorsorge vertraglich geregelt wurde. Die wichtigste Strategie war hierbei die Adoption einer Person, die den Adoptierenden pflegte und dafür nach dessen Ableben sein Erbe antrat. Daneben hatten Vermögende, die alleinstehend waren, die Möglichkeit die eigene Altersversorgung durch Kapitalbesitz zu sichern, sei es dadurch, dass man Sklaven die Freiheit garantierte, wenn sie die Alterspflege übernahmen, sei es dadurch, dass man Altenpflege durch Schuldentilgung einkaufte. Nur die besitz- und kinderlosen Alten waren auf die Barmherzigkeit anderer angewiesen. Manche Urkunden lassen erkennen, dass man mittellosen Alten 
in staatlichen und von Tempeln geführten Betrieben gegen Handlangerdienste ein mageres Altersbrot gewährte.

\section{Literaturverzeichnis}

Albertz R (1978) Hintergrund und Bedeutung des Elterngebots im Dekalog. Zeitschrift für Alttestamentliche Wissenschaft, 90:348-374

Arnaud D (1986) Recherches au pays d'Aštata. Emar VI.3. Textes sumériens et accadiens. Ed. Recherche sur les Civilisations, Paris

Böck B (2000) Die babylonisch-assyrische Morphoskopie. Archiv für Orientforschung Beiheft 27. Institut für Orientalistik der Universität Wien, Wien

Borger R (1982) Der Codex Hammurapi. In: Kaiser O (ed) Texte aus der Umwelt des Alten Testaments, Bd. I, Lieferung 1 (Rechtsbücher). Gütersloher Verlagshaus Mohn, Gütersloh, 39-80

Dandamaev MA (1980) About Life Expectancy in Babylonia. In: Alster B (ed) Death in Mesopotamia. Mesopotamica 8. Akademisk Vorlag, Kopenhagen, 183-186

David M (1927) Die Adoption im altbabylonischen Recht. Leipziger Rechtswissenschaftliche Studien 23 . Weicher, Leipzig

Farber W (1980-1983) Lamašhtu. In: Edzard DO u.a. (ed), Reallexikon der Assyriologie und Vorderasiatischer Archäologie. Sechster Band. de Gruyter, Berlin/New York, 439-446

Feix J (1963) Herodot. Historien. Griechisch-deutsch. Heimeran, München

Frahm E (2009) Historische und historisch-literarische Texte I, Keilschrifttexte aus Assur literarischen Inhalts 3. Wissenschaftliche Veröffentlichungen der Deutschen Orient-Gesellschaft 121. Harrassowitz, Wiesbaden

Geller MJ (2010) Ancient Babylonian Medicine: Theory and Practice. Wiley-Blackwell, Chichester

George AR (2003) The Babylonian Gilgamesh Epic. Introduction, Critical Edition and Cuneiform Texts, Oxford University Press, Oxford

Greenfield JC (1982) Adi baltu - Care for the Elderly and its Rewards. In: Hunger H, Hirsch H (ed) Vorträge gehalten auf der 28. Rencontre Assyriologique Internationale in Wien, 6.-10. Juli 1981. Archiv für Orientforschung Beiheft 19. Berger, Horn, 309-316

Gurney OR (1964) The Sultantepe Tablets II. British Institute of Archaeology at Ankara, London

Hecker K (1988) Die Adad-guppi-Inschrift. In: Kaiser O (ed) Texte aus der Umwelt des Alten Testaments, Bd II, Lieferung 4 (Grab-, Sarg, Votiv- und Bauinschriften). Gütersloher Verlagshaus Mohn, Gütersloh, 479-486

Heeßel NP (2000) Babylonisch-assyrische Diagnostik. Ugarit-Verlag, Münster

Lutzmann H (1982) Aus den Gesetzen des Königs Lipit Eschtar von Isin. In: Kaiser O (ed) Texte aus der Umwelt des Alten Testaments, Bd I, Lieferung 1 (Rechtsbücher). Gütersloher Verlagshaus Mohn, Gütersloh, 23-31

Malamat A (1982) Longevity: Biblical Concepts and Some Ancient Near eastern Parallels. In: Hunger $\mathrm{H}$, Hirsch $\mathrm{H}$ (ed) Vorträge gehalten auf der 28. Rencontre Assyriologique Internationale in Wien, 6.-10. Juli 1981. Archiv für Orientforschung Beiheft 19. Berger, Horn, 215-224

Maul SM (2007) Ringen um göttliches und menschliches Maß. Die Sintflut und ihre Bedeutung im Alten Orient. In: Hornung E, Schweizer A (ed), Schönheit und Mass. Beiträge der Eranos Tagungen 2005 und 2006. Schwabe, Basel, 161-183

ders. (2008) Das Gilgamesch-Epos. Vierte, durchgesehene Auflage. Beck, München

Mofidi Nasrabadi B (1999) Untersuchungen zu den Bestattungssitten in Mesopotamien in der ersten Hälfte des ersten Jahrtausends v. Chr. Baghdader Forschungen 23. von Zabern, Mainz

Neumann H (2003) Recht im antiken Mesopotamien. In: Manthe U (ed) Die Rechtskulturen der Antike. Vom Alten Orient bis zum Römischen Reich. Beck, München, 55-122

Parpola S (1993) Letters from Assyrian and Babylonian Scholars. State Archives of Assyria 10. Helsinki University Press, Helsinki 
Roth M (1987) Age at Marriage and the Household: A Study of Neo-Babylonian and Neo-Assyrian Forms. Comparative Studies in Society and History, 29:715-747

dies. (1991/93) The Neo-Babylonian Widow. Journal of Cuneiform Studies, 43-45:1-26

San Nicolò M (1932) Un contratto vitalizio del tempo di Dario I. Aegyptus 12:35-47

Schaudig H (2001) Die Inschriften Nabonids von Babylon und Kyros' des Großen samt den in ihrem Umfeld entstandenen Tendenzschriften: Textausgabe und Grammatik. Ugarit-Verlag, Münster

Stol M (1998) The Care of the Elderly in Mesopotamia in the Old Babylonian Period. In: Stol M, Vleeming SP (ed) The Care of the Elderly in the Ancient Near East. Brill, Leiden/Köln, 59-117 ders. (2000) Schwangerschaft und Geburt bei den Babyloniern und in der Bibel. In: Karenberg A, Leitz C (ed), Heilkunde und Hochkultur I. Geburt, Seuche und Traumdeutung in den antiken Zivilisationen des Mittelmeerraumes. Lit-Verlag, Münster, 97-115

Stol M, Vleeming SP (1998) The Care of the Elderly in the Ancient Near East. Brill, Leiden/Köln Tsukimoto A (1985) Untersuchungen zur Totenpflege (kispum) im alten Mesopotamien. Alter Orient und Altes Testament 216. Butzon \& Bercker, Kevelaer

Ungnad A (1908) Neubabylonische Kontrakte. Vorderasiatische Schriftdenkmäler 5. Hinrichs, Leipzig

van Driel G (1998) Care of the Elderly: The Neo-Babylonian Period. In: Stol M, Vleeming SP (ed) The Care of the Elderly in the Ancient Near East. Brill, Leiden/Köln, 161-197

Veenhof KR (1998) Old Assyrian and Ancient Anatolian Evidence for the Care of the Elderly. In: Stol M, Vleeming SP (ed) The Care of the Elderly in the Ancient Near East. Brill, Leiden/Köln, $119-160$

Westbrook R (1993) The Adoption Laws of Codex Hammurabi. In: Rainey, AF u.a. (ed) Kinattūtu ša dārâti. Raphael Kutscher Memorial Volume, TEL AVIV, Journal of the Institute of Archaeology of Tel Aviv University, Occasional Publications No. 1. University, Institute of Archaeology, Tel Aviv, 195-204

ders. (1998) Legal aspects of Care of the Elderly in the Ancient Near East: Introduction. In: Stol M, Vleeming SP (ed) The Care of the Elderly in the Ancient Near East. Brill, Leiden/Köln, $1-22$

ders. (2003) A History of Ancient Near Eastern Law. Brill, Leiden

ders. (2004) The Quality of Freedom in Neo-Babylonian Manumissions. Revue d'assyriologie et d'archéologie orientale, 98:101-108

Wilcke C (1984) Familiengründung im alten Babylonien. In: Müller EW (ed) Geschlechtsreife und Legitimation zur Zeugung. Alber, Freiburg (Breisgau), 213-317

ders. (1998) Care of the Elderly in Mesopotamia in the Third Millennium B.C. In: Stol M, Vleeming SP (ed) The Care of the Elderly in the Ancient Near East. Brill, Leiden/Köln, 23-57 